\title{
Special issue on ambient systems, networks and technologies
}

\author{
Ansar-Ul-Haque Yasar $^{1} \cdot$ Haroon Malik ${ }^{2}$ - Elhadi M. Shakshuki ${ }^{3}$
}

Published online: 31 October 2019

(C) Springer-Verlag London Ltd., part of Springer Nature 2019

This special issue is based on the best papers from 8th International Conference on Ambient Systems, Networks and Technologies (ANT-2017, http://cs-conferences.acadiau. ca/ANT-17/) and the 7th International Conference on Sustainable Energy Information Technology (SEIT 2017, http://cs-conferences.acadiau.ca/seit-17/) held in Madeira, Portugal, from May 16-19, 2017. Both conferences attracted a good number of scientific papers that contributed to the state-of-the-art in the areas of these conferences' themes. All the papers selected for this special issue have been extended from their original versions and underwent two rounds of rigorous peer-review process. Based on the reviewers' feedback, as well as the evaluations of the Guest Co-editors, 21 papers were selected for this special issue from 35 invited submissions. The accepted papers cover forthcoming development and emerging research along the lines of big data analytics, agent-based systems, IoT, WSNs and information systems.

One of the papers is by Igor et al. [1] entitled "Holonification Model for a Multilevel Agent-Based System. Application to Road Traffic". The authors of this paper presents a novel density approach to cluster and hierarchize population in order to build the initial holarchy. The proposal extends DBSCAN algorithm. Moreover, multilevel indicators based on standard deviation are proposed in order to evaluate

Ansar-Ul-Haque Yasar

ansar.yasar@uhasselt.be

Haroon Malik

malikh@marshall.edu

Elhadi M. Shakshuki

elhadi.shakshuki@acadiau.ca

1 Transportation Research Institute Hasselt University, Hasselt, Belgium

2 Weisberg Division of Computer Science, Huntington, WV, USA

3 Jodrey School of Computer Science Acadia University, Wolfville, Canada the consistency of the holonification process. The proposed model is tested in a road traffic modeling in order to build the initial holarchy. The paper presents also the main research direction toward the control of internal and external stimuli of traffic over time.

Another paper is by Ahmed et al. [2] entitled "Estimating pro-environmental potential for the development of mobilitybased informational intervention: a data-driven algorithm". This paper provides a methodological framework and describes the development of a computational algorithm that helps to identify soft changes in the travel behavior. The algorithm is based on a variety of different data sources such as activity-travel diaries and related constraint information, meteorological conditions, bicycle and public transport supply data, and emissions and air pollutant concentrations data. A variety of rules that are part of the algorithm are derived from the transport modeling literature, where constraints and factors were examined for activity-travel decisions. Three major aspects of activity-travel behavior, such as reduced car use, cold start of car engines, and participation in non-mandatory outdoor activities are considered in assessing pro-environmental potential. The algorithm is applied to collected small datasets from citizens of Hasselt (Belgium), Bologna (Italy), and Guildford (UK). A significant replaceable potential for car trips within $3 \mathrm{~km}$ to cycling and car trips to public transport has been found. The replaceable potential of excessive cold starts and participation in non-mandatory outdoor activities were also found, to some extent, to bring positive changes in the environment. In future research, these identified potentials are reported back to individuals with their consequence as part of a mobility-based informational intervention.

Another paper is by Jabeur et al. [3] entitled "A comprehensive reputation assessment framework for volunteered geographic information in crowdsensing applications". The authors provide as main contributions: (i) a multi-layer architecture for VGI which supports a process of reputation evaluation; (ii) a new comprehensive model for computing reputation scores for both VGI data and contributors, based on direct and indirect evaluations expressed by users, and including the 
concept of data aging; (iii) a variety of experiments evaluating the accuracy of the model. Finally, the relevance of adopting this framework is discussed via an applicative scenario for recommending tourist itineraries.

Another paper is by Leeuwen et al. [4] entitled "Network partitioning on time-dependent origin-destination electronic trace data". In this paper the authors identify spatial regions based on an empirical data set consisting of time-dependent origin-destination (OD) pairs. These OD pairs consist of electronic traces collected from smartphone data by Google in the Amsterdam metropolitan region and is aggregated by the volume of trips per hour at neighborhood level. By means of community detection, they examine the structure of this empirical data set in terms of connectedness.

Another paper is by Valmassoi et al. [5] entitled "Future Impacts of the Reforestation Policy on the atmospheric parameters in Ireland: a sensitivity study including heat discomfort impacts on humans and livestock". This study aims to assess the influence of the increase of vegetation cover over Ireland, on surface temperature, livestock, and human heat comfort, using the Weather Research Forecast (WRF-ARW 3.7.1) model. Multi-scale numerical simulations are performed under two scenarios: (i) a "control scenario" considering no change in vegetation cover with respect to the prescribed one and (ii) a "green scenario" with increased tree cover based on the introduced Irish Reforestation policy. To simulate this policy, the cropland and vegetative mosaic is substituted with evergreen broad-leaf forest, increasing the total forest area from 19.7 to $36.2 \%$ of the land in the analyzed domain. This change in vegetation cover increases the temperature over the simulated domain up to $0.7^{\circ} \mathrm{C}$ and, moreover, it enhances both human and livestock heat discomfort during the daytime, with different magnitude all over the domain. It is concluded that the reforestation policy, which is introduced to mitigate the climate warming and greenhouse gas emissions, causes a further increase in temperature along with heat discomfort to both human and livestock.

Another paper is by Miao et al. [6] entitled "Optimal secret sharing for wireless information security in the era of Internet of Things". In this paper the authors study how to optimally share secrets between two and more users using the effect of wireless channel dynamics on the data link layer. For the two-user case, they formulate an optimization problem whose objective is to minimize the expectation of the probability that an eavesdropper receives all secret sharing packets. Their contributions are as follows: (i) they come up with the secret sharing mechanism that minimizes the aforementioned objective function and (ii) they perform analysis on our approach and derive the worst-case probability of the eavesdropper receiving all secret sharing packets.

Another paper is by Braun et al. [7] entitled "Investigating large curved interaction devices". In this paper the authors present Curved, a large-surface interaction device, whose shape is designed based on the natural movement of an outstretched arm. It is able to track one or two hands above or on its surface by using 32 capacitive proximity sensors. Supporting both touch and mid-air interaction can enable more versatile modes of use. They use image processing methods for tracking the user's hands and classify gestures based on their motion. Virtual reality is a potential use case for such interaction systems and was chosen for their demonstration application. They conducted a study with 10 users to test the gesture tracking performance, as well as user experience and user preference for the adjustable system parameters.

Another paper is by Arsalan et al. [8] entitled "Semantic enrichment of spatio-temporal trajectories for worker safety on construction sites". The authors of this paper proposed a system to monitor worker movements on a construction site by collecting their raw spatio-temporal trajectory data and enriching it with the relevant semantic information. To acquire the trajectories, the use of an indoor positioning system (IPS) is considered. Bluetooth beacons are used for collecting spatio-temporal information of the building users. By means of an Android-based mobile application, neighboring beacons' signals are selected, and a geo-localization technique is performed to get the unique pairs of users' location coordinates. After pre-processing this collected data, three semantic enrichment techniques are used to construct semantically enriched trajectories.

Another paper is by Souidi et al. [9] entitled "Geographic forwarding rules to reduce broadcast redundancy in mobile ad hoc wireless networks". The authors propose a new decentralized technique, called geographic forwarding rules (GFRs), to reduce the number of broadcast messages in mobile ad hoc networks. They use the location information of nodes to divide the network into virtual zones. Then they try to avoid duplicate retransmissions between the zones. Their proposition reduces the amount of overhead while it achieves a successful dissemination.

Another paper is by Al-Obeidat et al. [10] entitled "Hybrid multicriteria fuzzy classification of network traffic patterns, anomalies, and protocols". In this paper the authors propose a new supervised hybrid machine-learning approach for ubiquitous traffic classification based on multicriteria fuzzy decision trees with attribute selection. Moreover, our approach can handle well the imbalanced datasets and zero-day applications (i.e., those without previously known traffic patterns). Evaluating the proposed methodology on several benchmark real-world traffic datasets of different nature demonstrated its capability to effectively discriminate a variety of traffic patterns, anomalies, and protocols for unencrypted and encrypted traffic flows. Comparing with other methods, the performance of the proposed methodology showed remarkably better classification accuracy.

Another paper is by Mbarek et al. [11] entitled "ECASS: an encryption compression aggregation security scheme for secure data transmission in ambient assisted living systems". 
The authors propose in this paper to combine the use of cryptography, compressed sensing, and steganography into a new generic solution called Encryption Compression Aggregation Security Scheme (ECASS). While focusing on the specific case of Medical Healthcare Systems, ECASS aims to secure private data exchanges over wireless networks while achieving lower energy consumption and communication overhead. Their simulations with the NS-2.35 simulator are showing an improvement of 40 and $50 \%$ in terms of energy consumption and communication overhead respectively compared to the IBE-Lite security scheme.

Another paper is by Yacchirema et al. [12] entitled "Fall detection system for elderly people using IoT and ensemble machine learning algorithm". In this paper the authors propose IoTE-Fall system, an intelligent system for detecting falls of elderly people in indoor environments that takes advantages of the Internet of Thing and the ensemble machine learning algorithm. IoTE-Fall system employs a 3D-axis accelerometer embedded into a 6LowPAN wearable device capable of capturing in real time the data of the movements of elderly volunteers. To provide high efficiency in fall detection, in this paper, four machine learning algorithms (classifiers): decision trees, ensemble, logistic regression, and Deepnets are evaluated in terms of AUC ROC, training time and testing time.

Another paper is by Darabkh et al. [13] entitled "AEAFCP: an adaptive energy-aware fixed clustering protocol for data dissemination in wireless sensor networks". In this paper the authors propose multiple novel mechanisms to achieve this goal. Firstly, a new approach is presented for the construction of clusters with balanced size and even distribution during the initial cluster head selection. Secondly, a novel scheme is introduced for distributing the cluster head task, dependent upon a node's energy and location information within each cluster. Lastly, a multi-hop routing paradigm is employed to minimize the communication distances and save the nodes' energy. The results of the simulation showed that our protocol's performance surpasses other directly connected works in both continuous data and event-based applications.

Another paper is by Al-Ruithe et al. [14] entitled "A systematic literature review of data governance and cloud data governance". In this paper the authors propose a systematic literature review (SLR), which offers a structured, methodical, and rigorous approach to the understanding of the state-of-theart of research in data governance. The objective of the study is to provide a credible intellectual guide for upcoming researchers in data governance to help them identify areas in data governance research where they can make the most impact. The systematic literature review covered published contributions from both academia and industry. The presented SLR searches and examines most relevant published work since year 2000 to-date for data governance for non-cloud, and for cloud computing since 2007. Only 52 studies met the inclusion criteria, which are critically reviewed.
Another paper is by Elbes et al. [15] entitled "Using phase shift fingerprints and inertial measurements in support of precise localization in urban areas". In this paper the authors present a particle filter-based data fusion technique for localization in urban areas. The proposed localization technique provides more accurate location estimation results due to its ability to efficiently fuse together information collected from diverse sensor technologies. The novelty of their proposed approach stems from its ability to fuse data from diverse sources, namely, phase shift fingerprints collected from Low Power AM Radio (LPAM) towers and inertial measurement sensors.

Another paper is by D'Ambra et al. [16] entitled "Affordance Theory and E-Books: Evaluating the E-reading Experience Using Netnography". E-books have changed the business of book publishing and the reading experience of the general public. Dedicated e-readers and other smart devices are integral to ebooks offering affordances to overcome the physical limitations of print book and to provide the functionality of information technology. Using netnography, comments by online readers to articles in The New York Times and Scientific American were analyzed and coded by themes identified in the literature of ebooks versus print books. An Affordance Theory approach was used to provide insights into the readers' perceptions of real and actual affordances and the value delivered by these affordances. Comments by online readers of two diverse datasets confirm results found in questionnaires and surveys reported in the academic literature. It is the physical attributes and functionality of smart devices used in e-reading that provides the opportunity of affordance. Their study provides support for an affordance perspective of e-books and e-readers. It also highlights preferences for e-booksand/or print books in various contexts. To our knowledge it is the first to consider e-readers as an IT artifact providing information processing capabilities.

Another paper is by Outay et al. [17] entitled "Investigation of the impact of a wireless Fog Warning System with respect to road traffic on a highway". In this paper the authors present a Wireless Fog Warning System (WFWS) where cooperative awareness messages are disseminated and used for calculating acceleration/deceleration activities. Without loss of generality, we build on IEEE 802.11p WLAN as a basis technology. Using simulations on the open-source vehicular network simulation framework Veins, we demonstrate both the potential of such a system for increasing safety and smoothing traffic flow-as well as of computer simulation as a means of its evaluation.

Another paper is by Salem et al. [18] entitled "Enhanced LEACH protocol for increasing a lifetime of WSNs". The aim of this paper is to overcome these limitations through enhancing the LEACH (low energy adaptive clustering hierarchy) protocol, the protocol of cluster routing, in which, LEACH is extended by identifying a cluster head according to the lowest degree of distance from the base station in order to 
decrease power consumption in cluster head nodes and in the whole network. Hence, the results clarify the ability of LEACH in enhancing the network lifetime as well as in reducing and minimizing the consumption of power.

Another paper is by Bouyahia et al. [19] entitled "A twostage road traffic congestion prediction and resource dispatching toward a self-organizing traffic control system". In this paper the authors present a new two-stage traffic resource dispatching solution which is ultimately aiming to implement a self-organizing traffic control system based on Internet of Things. Their solution uses in its first phase a Markov Random Field (MRF) to model and predict the spread of traffic congestions over a road network. According to the obtained predictions, the solution uses Markov Decision Process (MDP) to automatically allocate the road traffic resources. Their simulations are showing satisfactory results in terms of efficient intervention ratios compared to other solutions.

Another paper is by Spencer et al. [20] entitled "Accurately forecasting temperatures in smart buildings using fewer sensors". In this paper the authors besides the cost of energy, consider the cost of data collection and management. Their approach informs the selection of an optimal set of sensors for any model predictive controller to reduce overall costs, using any forecasting methodology. They use lasso regression with lagged observations, which compares favorably to previous methods using the same data.

Another paper is by Riaz et al. [21] entitled "Evaluation of a gamified e-learning platform to improve traffic safety among elementary school pupils in Belgium". Children are highly represented in statistics relating to road traffic injuries and fatalities. There are multiple risk factors that together create an increased risk for children in traffic, some of the major reasons include children's inability to efficiently and actively scan the environment and look for information relevant to the traffic environment, inconsistent behavior, and less developed hazard perception skills. Traffic safety education is one of the most important means for improving knowledge and skills required for children to behave safely in traffic. This study evaluated a newly developed and gamified e-learning platform meant to promote traffic safety among elementary school pupils in Belgium. Participants in this study were from four grades of elementary school and voluntarily took part in the training. They followed a self-study program at home for approximately $15 \mathrm{~min}$ per week over a period of 5 weeks in total. The platform included four modules: traffic knowledge, situation awareness, risk detection, and risk management. For each of these modules, a set of photos and videos were used as stimuli and selected from a database of camera recordings of real-life situations. Half of each module consisted of familiar situations for the pupils (i.e., own municipality), while the other half of each module consisted of unfamiliar situations for the pupils (i.e., other municipalities). A fifth module, "the finale," contained a mix of the first four modules. In total, 44 elementary school pupils (9-13 years old) completed the program. During the first round of measurement (i.e., the first four modules), pupils performed significantly better in the traffic knowledge module when compared with the other three modules. Further, in comparison with unfamiliar situations, pupils scored significantly higher in familiar situations. During the second round of measurement (i.e., the fifth module), pupils achieved higher scores in the risk detection and risk management modules when contrasted to the first measurement. The effect of gamification elements is discussed and the results also indicate the type of traffic safety issues to be emphasized in traffic safety education for children.

Another paper is by Banerjee et al. [22] entitled "Superposition modulation-based new structure of four-dimensional turbo code (4D-TC) using modified interleaver and its application in WiMAX \& LTE systems". In this paper a modified interleaver-driven binary 4-Dimensional Turbo Code (4D-TC) using superposition modulation (SM) technique has been projected to intensify the minimum hamming distance (MHD). MHD (dmin) of the proposed structure has been amplified by incorporating feedback polynomial in primitive form for a fixed interleaver length. Moreover, improved dmin has further been achieved by introducing a modified interleaver which has been used to ensure scattering and mixing operation on the incoming bits in an appropriate manner.

The guest editors would like to take this opportunity to thank all the authors for the efforts they put in the preparation of their manuscripts and for their valuable contributions. We wish to express our deepest gratitude to the referees, who provided useful and constructive feedback to the authors. Our sincere thanks go to the Editor-in-Chief for his kind help and support.

\section{References}

1. Igor TH, Galland S, Kamla V, Kamgang JC, Saha C, Zhao H (2018) Holonification model for a multilevel agent-based system. Application to Road Traffic Personal and Ubiquitous Computing. https://doi.org/10.1007/s00779-018-1181-y

2. Ahmed S, Adnan M, Janssens D, Brattich E, Yasar A-U-H, Kumar P, Di Sabatino S, Shakshuki E (2018) Estimating proenvironmental potential for the development of mobility-based informational intervention: a data-driven algorithm. Pers Ubiquit Comput. https://doi.org/10.1007/s00779-018-1187-5

3. Jabeur N, Karam R, Melchiori M, Renso C (2018) A comprehensive reputation assessment framework for volunteered geographic information in crowdsensing applications. Personal and Ubiquitous Computing 1-17. https://doi.org/10.1007/s00779-018-1122-9

4. Leeuwen D, Bosman J, Dugundji E (2019) Network partitioning on time-dependent origin-destination electronic trace data. Pers Ubiquit Comput. https://doi.org/10.1007/s00779-019-01208-1

5. Valmassoi A, Gharbia S, Di Sabatino S, Kumar P, Pilla F (2018) Future impacts of the reforestation policy on the atmospheric parameters in Ireland: a sensitivity study including heat discomfort 
impacts on humans and livestock. Pers Ubiquit Comput. https://doi. org/10.1007/s00779-018-1160-3

6. Miao L, Jiang D (2019) Optimal secret sharing for wireless information security in the era of internet of things. Pers Ubiquit Comput. https://doi.org/10.1007/s00779-019-01226-z

7. Braun A, Zander-Walz S, Majewski M, Kuijper A (2018) Investigating large curved interaction devices. Pers Ubiquit Comput. https://doi.org/10.1007/s00779-018-1109-6

8. Arslan M, Cruz C, Ginhac D (2019) Semantic enrichment of spatiotemporal trajectories for worker safety on construction sites. Pers Ubiquit Comput. https://doi.org/10.1007/s00779-018-01199-5

9. Souidi M, Habbani A, Halim B, El Mahdi F (2018) Geographic forwarding rules to reduce broadcast redundancy in mobile ad hoc wireless networks. Pers Ubiquit Comput. https://doi.org/10.1007/ s00779-018-1137-2

10. Al-Obeidat F, El-Alfy E-S (2017) Hybrid multicriteria fuzzy classification of network traffic patterns, anomalies, and protocols. Pers Ubiquit Comput. https://doi.org/10.1007/s00779-017-1096-z

11. Mbarek B, Jabeur N, Yasar A-U-H(2018) ECASS: an encryption compression aggregation security scheme for secure data transmission in ambient assisted living systems. Pers Ubiquit Comput. https://oi.org/10.1007/s00779-018-1128-3

12. Yacchirema D, Puga J, Palau C, Esteve M (2019) Fall detection system for elderly people using IoT and ensemble machine learning algorithm. Pers Ubiquit Comput. https://doi.org/10.1007/s00779018-01196-8

13. Darabkh K, Al-Jdayeh L (2019) AEA-FCP: an adaptive energyaware fixed clustering protocol for data dissemination in wireless sensor networks. Pers Ubiquit Comput. https://doi.org/10.1007/ s00779-019-01233-0

14. Al-Ruithe M, Benkhelifa E, Hameed K (2018) A systematic literature review of data governance and cloud data governance. Pers Ubiquit Comput. https://doi.org/10.1007/s00779-017-1104-3
15. Elbes M, Alkhatib A, Al-Fuqaha A, Qadir J (2019) Using phase shift fingerprints and inertial measurements in support of precise localization in urban areas. Pers Ubiquit Comput. https://doi.org/10. 1007/s00779-019-01227-y

16. D'Ambra J, Wilson C, Akter S (2017) Affordance theory and Ebooks: evaluating the E-reading experience using netnography. Pers Ubiquit Comput. https://doi.org/10.1007/s00779-017-1086-1

17. Outay F, Ahmar A, Kamoun F, Yasar A-U-H, Sommer C, Jabeur N, El-Amine S (2018) Investigation of the impact of a wireless fog warning system with respect to road traffic on a highway. Pers Ubiquit Comput. https://doi.org/10.1007/s00779-018-1151-4

18. Abu Salem A, Shudifat N (2019) Enhanced LEACH protocol for increasing a lifetime of WSNs. Pers Ubiquit Comput. https://doi. org/10.1007/s00779-019-01205-4

19. Bouyahia Z, Haddad H, Jabeur N, Yasar A (2019) A two-stage road traffic congestion prediction and resource dispatching toward a selforganizing traffic control system. Pers Ubiquit Comput 1-12. https://doi.org/10.1007/s00779-019-01212-5

20. Spencer B, Al-Obeidat F, Alfandi O (2017) Accurately forecasting temperatures in smart buildings using fewer sensors. Pers Ubiquit Comput. https://doi.org/10.1007/s00779-017-1103-4

21. Riaz MS, Cuenen A, Janssens D, Brijs K, Wets G (2019) Evaluation of a gamified e-learning platform to improve traffic safety among elementary school pupils in Belgium. Pers Ubiquit Comput. https://doi.org/10.1007/s00779-019-01221-4

22. Banerjee, Subhabrata, Chattopadhyay, Sudipta (2019) Superposition modulation-based new structure of four-dimensional turbo code (4D-TC) using modified interleaver and its application in WiMAX \& LTE systems. Personal and Ubiquitous Computing. https://doi.org/10.1007/s00779-019-01295-0

Publisher's note Springer Nature remains neutral with regard to jurisdictional claims in published maps and institutional affiliations. 